\title{
Procedural Techniques of Coronary Angiography
}

\author{
Jasmin Čaluk \\ BH Heart Center Tuzla \\ Bosnia-Herzegovina
}

\section{Introduction}

Coronary angiography is an invasive diagnostic procedure in which radiocontrast is injected into the coronary arteries under X-ray guidance in order to display the coronary anatomy and possible luminal obstruction. Despite the advances in other diagnostic methods, it remains to be "the golden standard" of coronary disease diagnostics. Although today the complication rate is far lower than previously, the possibility of complication still exists, and an invasive cardiologist must be able to complete the procedure flawlessly, and to competently deal with complications, should they occur. In order to be able to do that, he/she must master the proper techniques in performing the coronary angiography procedure, and be comfortable with all the available access-sites.

\section{Cath lab}

The procedure is performed in a specifically designed room, very much resembling the operating room, called the catheterization laboratory, or cath lab (Fig.1). Recordings are made using a special X-ray machine, called cardioangiograph (Fig.1). Today, these machines are completely digitalized, enabling superb picture quality, with the lowest possible radiation doses, and some additional features, such as precise measurements and quantifications of the recorded angiograms, connections to additional devices, such as intravascular ultrasound, easy and large-capacity data storage etc. It is convenient to have a wide choice of spare catheters, multi-packs, guidewires, coronary angioplasty ballons, coronary stents, and other equipment in the cath lab itself, so it can be used without time-delay. The space should be intuitively organized, and the spare devices, sterile gowns, gloves, drapes etc. must be easily accesible. Defibrillator, endotracheal tubes, temporary pacemakers and leads, oxygen supply, and other cardiopulmonary resuscitation equipment must be held in the cath lab at all times. Just outside of the cath lab is the control-station with monitors for angiogram, patient data, electrocardiogram (ECG), haemodynamics etc (Fig.2). Modern cardioangiographs allow for data analysis both from the control station outside the cath lab, as well as from within the cath lab, using specifically designed control-commands at the table.

\section{Coronary angiography: Preparation}

Coronary angiography is performed under local anesthesia. The procedure is sterile, and all potential access sites must be disinfected, shaved, and sterilized. At the beginning of the 
procedure, the patient lays down in supine position on the cardioangiograph table, and is prepared for the procedure in sterile conditions (Fig.3).

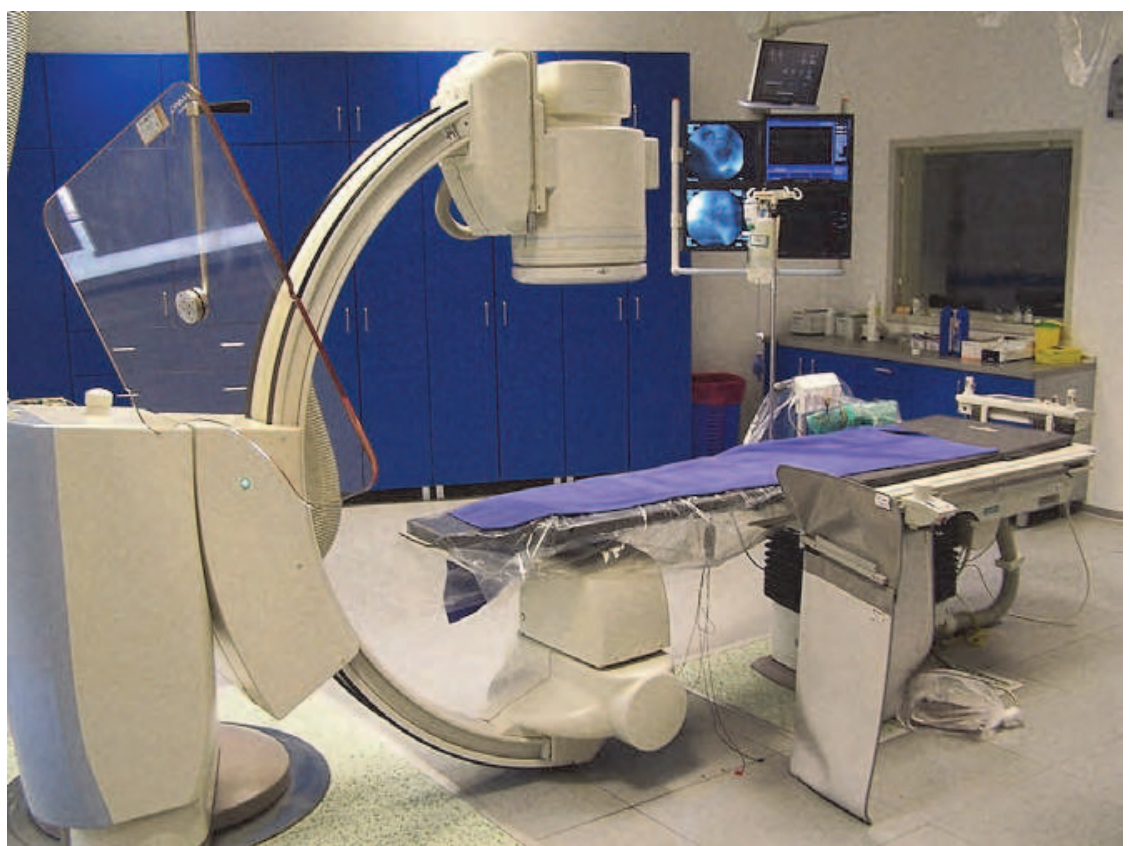

Fig. 1. Catheterization laboratory

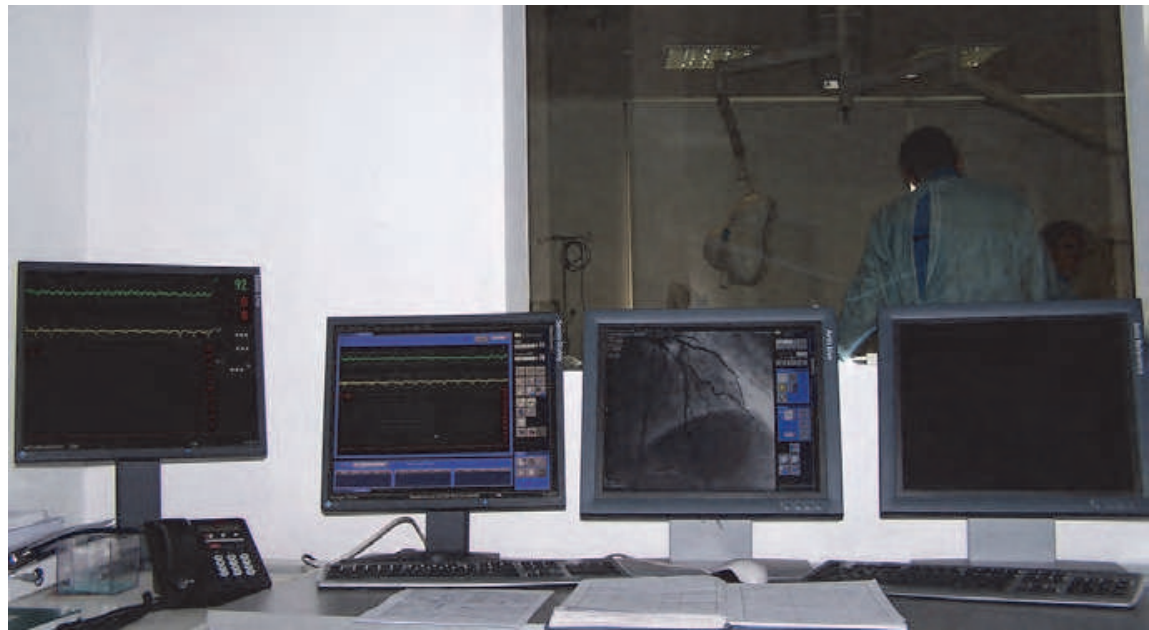

Fig. 2. Control-station with monitors outside the catheterization laboratory 


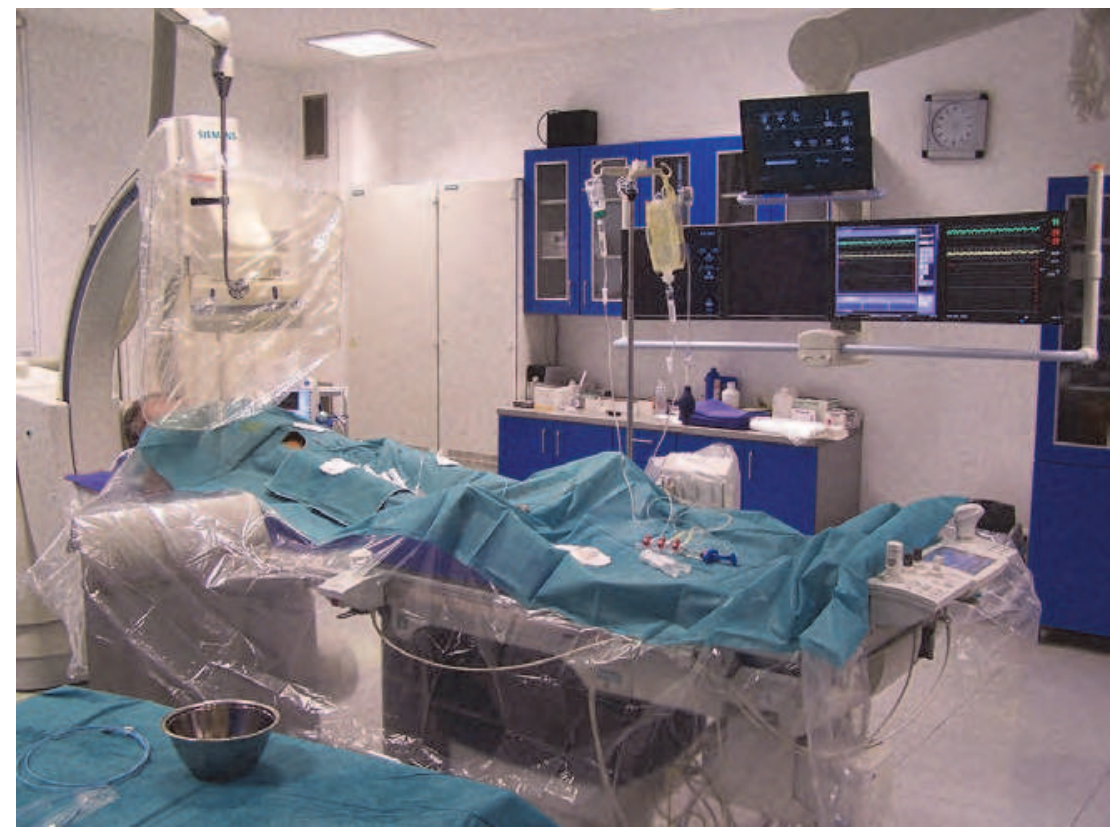

Fig. 3. The patient in supine position on the cardioangiograph table

Coronary angiography is performed with the patient in the fasting state, except in emergency cases, e.g. acute myocardial infarction, in which the degree of urgency prevails over the risks of aspiration incidents, which are generally very rare. In many institutions today, it is common not to administer pre-medication prior to coronary angiography, except in certain specific conditions. In other institutions, the patients are given $10 \mathrm{mg}$ diazepam p.o. and $25 \mathrm{mg}$ diphenhydramine p.o. 30-60 $\mathrm{min}$ before the procedure. If the patient has a known allergy to radiocontrast, prednisone can be administered at $60 \mathrm{mg}$ p.o. every 8 hours for two days prior to the procedure. Other institutions have the policy to administer hydrocortisone $80-120 \mathrm{mg}$ IV some $30 \mathrm{~min}$ before the procedure, along with $50 \mathrm{mg}$ diphenhydramine p.o. The patients with a history of radiocontrast allergy must be monitored after coronary angiography for signs of acute allergy: urticas on skin or itchy skin, swelling of tongue, stuffy nose or signs of respiratory distress.

The table set for coronary angiography (Fig. 4) can differ from one institution to another, but generally it has the following list of items and equipment:

- sterile drapes to cover the patient and radiation-protection screens,

- sterile swabs for cleaning the arterial access site,

- a bowl of Betadine solution for cleaning the arterial access site,

- lidocaine for local anesthesia, $15-20 \mathrm{ml}$ of $2 \%$ solution (in some cases, for more sensitive patients, more may be needed)

- punction neelde for arterial cannulation,

- $\quad$ an 11-mm or 15-mm blade,

- $\quad$ syringes and needles,

- two containers, one with $500 \mathrm{ml}$ of saline solution with 5,000 U of heparin added,

- $\quad$ arterial sheath with a dilator, 
- a J-shaped guidewire,

- diagnostic catheters for coronary angiography; start with the most common JL4 and JR4 with angulated pigtail if left ventriculography and/or aortography is planned,

- a three-outlet manifold which is to be connected to pressure transducer, saline solution container, and radiocontrast container on side-outlets, to the syringe for radiocontrast application on the proximal end, and with its distal end to diagnostic/therapeutic catheters during the procedure.

During the procedure, the patient must be monitored by several systems, and the ECG is absolutely essential. The patient also must have an ECG recording done just before the procedure actually starts. For practical reasons, during the angiography itself, there is really no need to apply the precordial leads, since the basic four electrodes can provide enough information for ECG monitoring during coronary angiography and percutaneous coronary interventions (PCI). Four electrodes should be placed on the calf or thigh and on the shoulders or upper arms (Watson \& Hunter, 2011).

The area of the puncture site must be shaved, disinfected, then anesthetized, and only then punctured. The center of the field for shaving and disinfection is the puncture site. The disinfection is performed starting at the center, and spiraling outwards, at least twice, using Betadine. Thereafter, the site is wiped off with a third swab. If the procedure is planned to be performed via the femoral artery, than both right and left groins must be shaved and disinfected, in case that the operator must switch to contralateral artery. Only after the disinfection of the puncture site, the patient is covered with a sterile drape. Most centers today use single-use pre-packed sterile drapes. These drapes have openings conveniently placed at the location of patient's left and right inguinal area (Fig.5, Fig. 6).

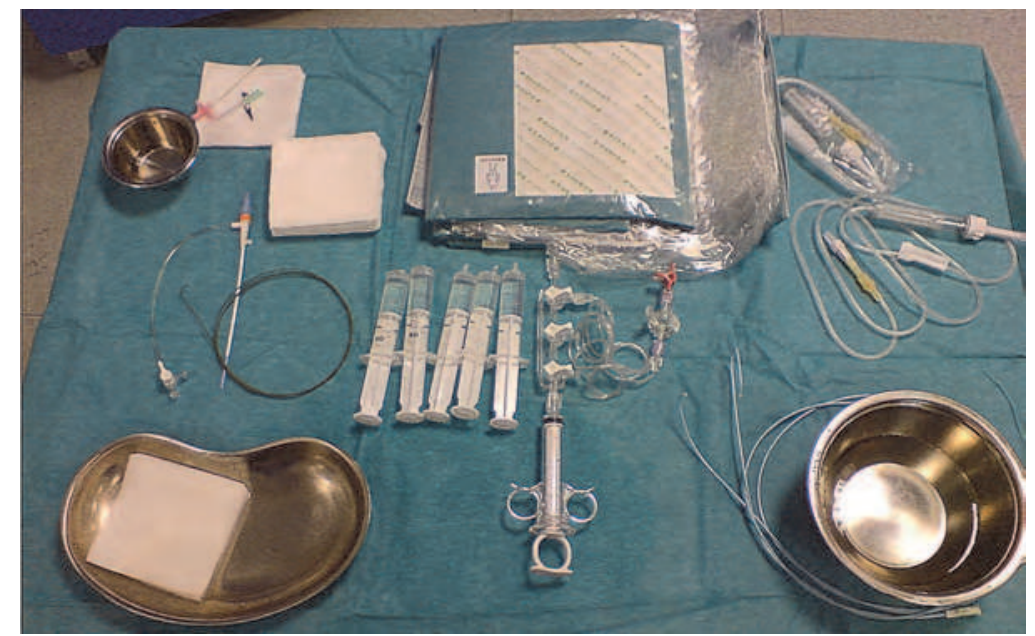

Fig. 4. The table set for coronary angiography

The patient must be warned not to move his/her hands downward, towards the disinfected areas of planned vascular access. The radiation shield and image intensifier/digital flat panel detector are also covered with sterile covers. The manifold is connected and flushed, and then zeroed for pressure monitoring. 


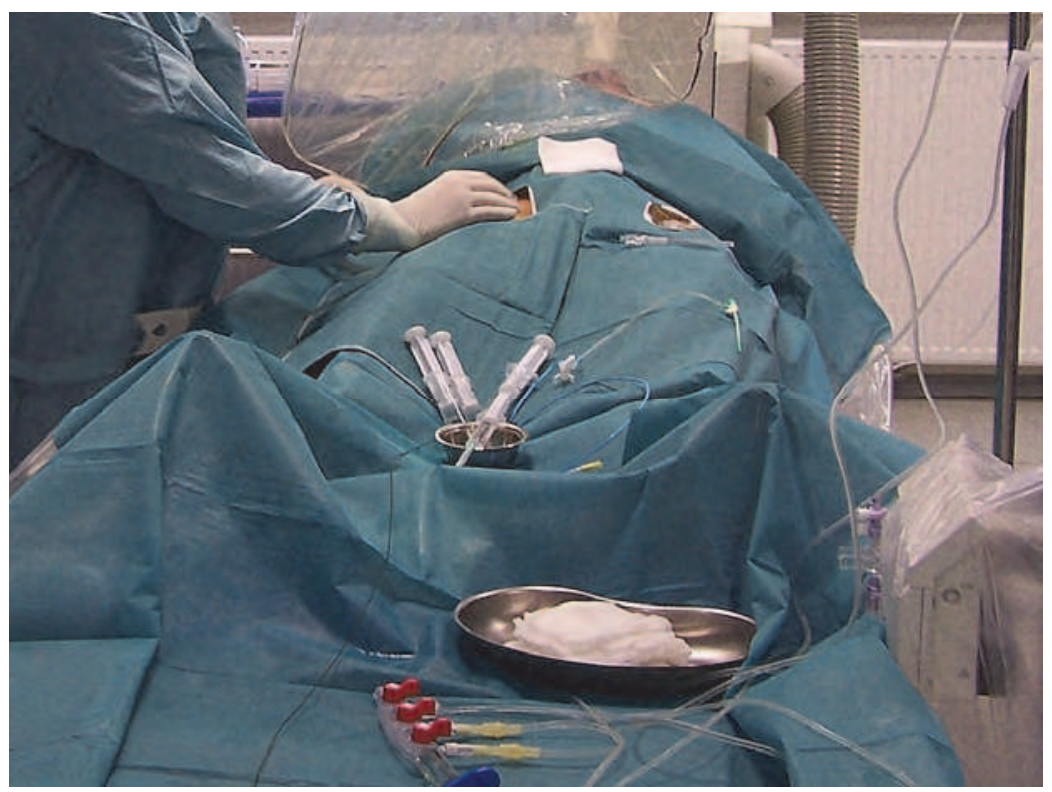

Fig. 5. Sterile drape openings in patient's inguinal area, femoral pulse palpation

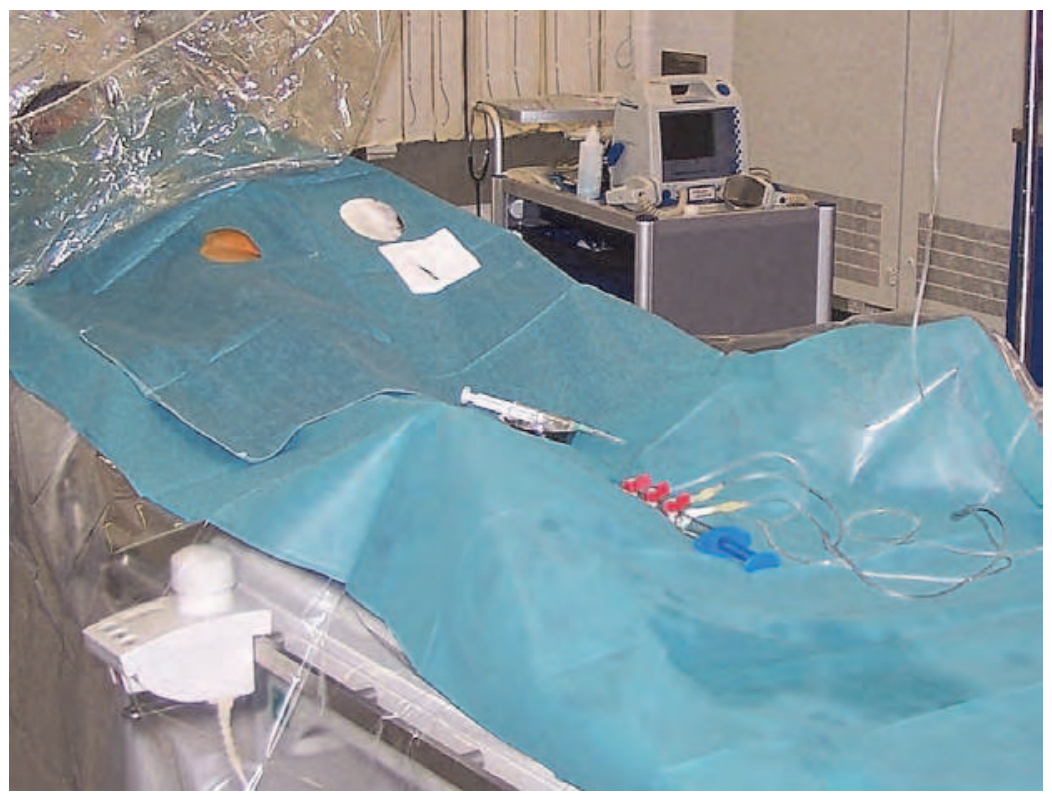

Fig. 6. Sterile drape openings in patient's inguinal area 


\section{Catheter menu}

Coronary angiography is performed with the use of specifically designed diagnostic catheters. More than $95 \%$ of all procedures can be completed using catheters Judkins left 4 (JL4), and Judkins right 4 (JR4). Also, a pigtail catheter is used for obtaining left ventriculogram, if it is recorded. Usually the catheters come pre-packed in a sterile envelope, in which JL4, JR4 and pigtail catheters are present. Diagnostic catheters are more elastic than the catheters used for PCI, and can be manipulated into the coronary ostium. However, sometimes the anatomy of the aortic arch and coronary ostia requires the use of different types of catheters. Today, the catheter menu is abundant with different versions, and at least one of the available catheters will be able to adequately cannulate even the most difficult and distorted coronary ostia (Fig.7). It is necessary to know the shapes of those catheters in order to choose the appropriate-ones for certain anatomical variants. Therapeutic (guiding) catheters are somewhat stiffer and do not forgive an imperfect position, because the insertion of interventional equipment (coronary guidewires, angioplasty balloons, stents etc.) creates much more backforce than does the radiocontrast injection. Therefore, an unstable catheter with inadequate backup will be frequently displaced out of the coronary ostium. This is why coronary stenting, even more than diagnostic coronary angiography, requires the use of dedicated guiding catheters.

\section{Arterial puncture}

The standard technique of arterial puncture used today is Seldinger technique (Powell \& Moxey, 2011), cleverly developped by Sven-Ivar Seldinger, a Swedish radiologist, in 1953. The procedure of arterial puncture is performed as follows: firstly, at the site of arterial puncture, an appropriate pulse has to be palpated in order to locate the artery (Fig.5). A local anesthesia is applied, usually with $10-15 \mathrm{ml}$ of $1 \%$ or $2 \%$ lidocaine for local infiltration of the skin and subcutaneous tissues. Then, a percutaneous puncture of the desired vessel with a hollow beveled tapered-tip needle is made. The pointy tip at the distal end of the needle is the first one in contact with the skin, and subsequently with the artery, while the distal needle opening faces upwards. The needle is held in the operator's hand like a pencil, at an angle of $30^{\circ}-45^{\circ}$ against the surface of the skin. Arterial puncture is made by advancing the needle to the site of the arterial pulse. An experienced operator will usually feel when the needle has punctured the arterial wall. A correct arterial puncure is confirmed by the pulsatile back-flow of the bright-red blood from the proximal end of the needle (Fig.8).

It is not necessary to have a syringe attached to the needle during arterial puncture, because the arterial pressure will produce a pulsatile jet of blood when the needle tip reaches the arterial lumen. If the backflow is slow and barely pulsatile (or seems to be continuous, without pulsations), and the blood is dark-red, the needle tip has punctured the vein. In certain circumstances, however, even correct arterial puncture may resemble venous puncture: shock, hypotension, severe bradycardia, very low oxygen saturation, cardiac arrest and/or resuscitation etc.

After the arterial puncture, an atraumatic, curved-tipped guide wire (J-wire with moveable inner core) is passed through the needle and advanced into the artery (Fig.9). The needle is then removed, leaving the guide wire in the vessel. The site of puncture at the skin level is usually widened by a couple of milimetres using a small and shallow cut by a surgical scalpel, an 11-mm or 15-mm blade to facilitate the passage of the arterial sheath. The sheath 
with a plastic dilator inside of it is advanced over the guide wire, and into the artery. The dilator is removed, leaving the sheath in the vessel, and a haemostatic valve prevents bleeding through the sheath. It also provides access for the passage of catheters and other devices into the vessels, with no blood loss. The side arm of the sheath is aspirated and injected with heparinized saline before the advancement of catheters. During arterial puncture and sheath placement, the patient may develop a vasovagal reaction due to puncture and vessel manipulation, which will manifest as hypotension and bradycardia. Atropine IV usually swiftly reverts the condition, and sometimes elevation of patient's legs and high-rate saline infusion with oxygen supplementation should be implemented additionally. The technique of direct arterial approach, with the preparation and direct visualization of the artery at the puncture site is today very rarely used, and therefore discussed elsewhere.

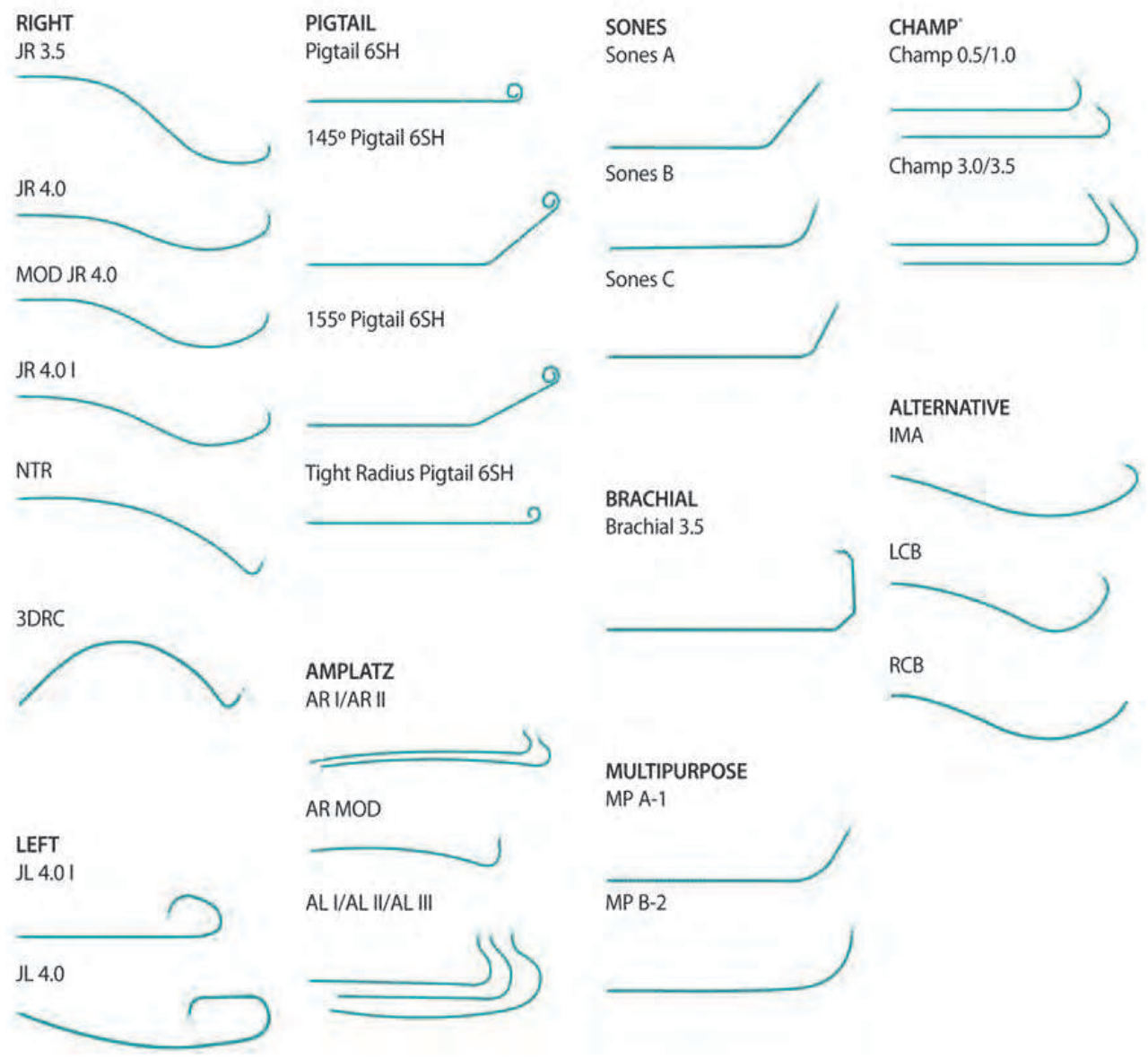

Fig. 7. Diagnostic catheter curves, picture courtesy of Medtronic 


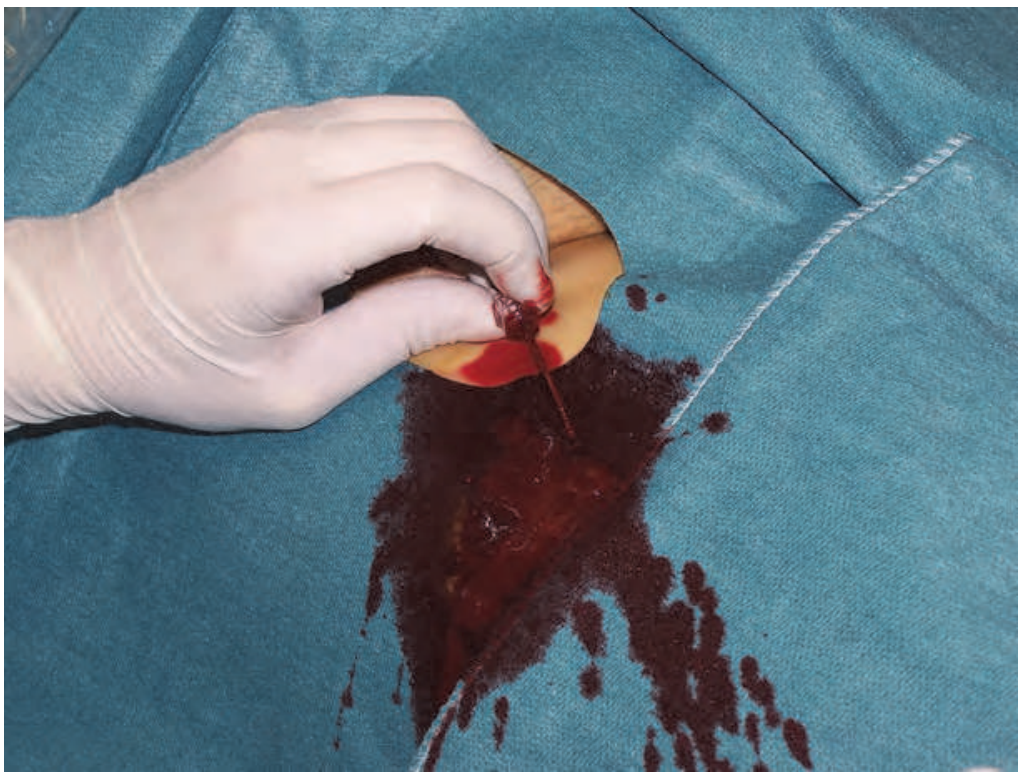

Fig. 8. Pulsatile back-flow of the bright-red blood from the proximal end of the needle

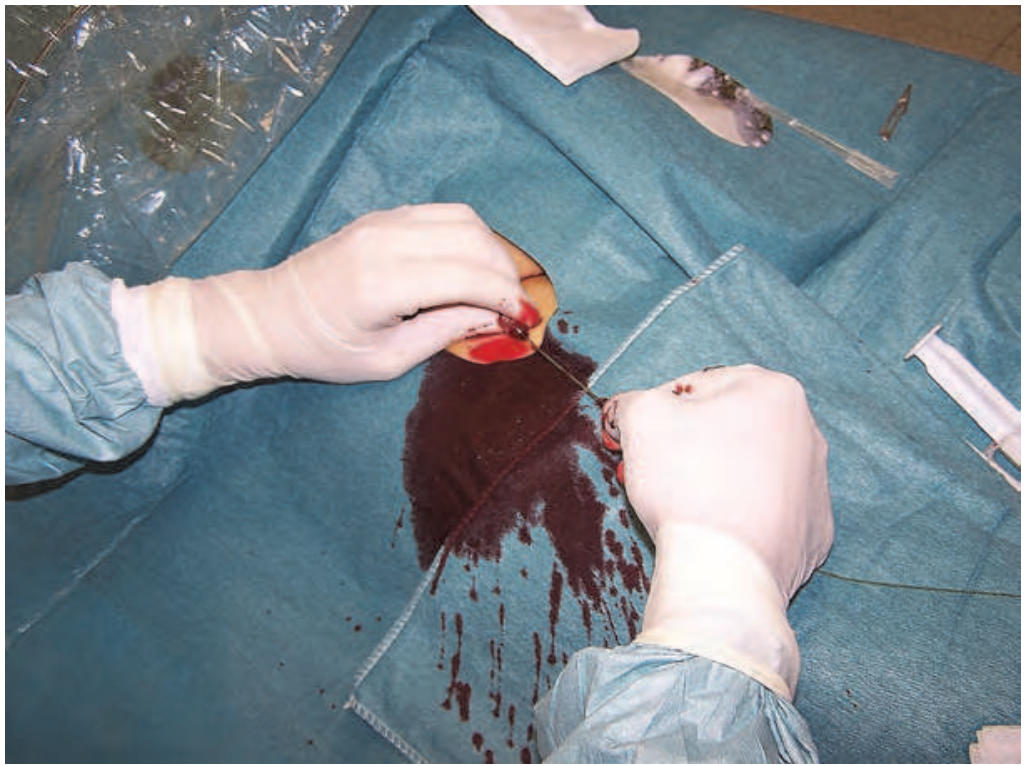

Fig. 9. J-wire is passed through the needle and advanced into the artery 


\section{Vascular access}

\subsection{Femoral approach}

Preferences of particular access sites vary in different centers, but the most commonly used are right femoral and right radial artery. The percutaneous transfemoral approach has been routinely used for cardiac catheterization and coronary angioplasty (Kwac et al, 2010). Right transfemoral approach is favoured because of the larger artery diameter, and therefore the possibility to insert larger arterial sheaths, catheters and bulkier devices, in comparison with the transradial approach. If there is a peripheral vascular problem, the left femoral, or right or left radial or brachial approaches can be used. The femoral approach is the most comfortable access for the operator. Its main disadvantage is the time-delay after the angiography and before mobilizing the patient. After the femoral pulse is palpated (Fig.5) and local anesthesia applied, the puncture of the femoral artery is performed some $2 \mathrm{~cm}$ below the inguinal ligament (Fig.10), at the punctum maximum of the pulse. The inguinal ligament is attached to spina illiaca anterior superior on one end, and with the pubic bone on the other (Fig.10). This makes determining of its anatomical location easier, because overlapping skin crease in obese patients can be located more distally, thus deceptively making the less experienced operators prone to puncturing too distally. Puncture through the ligament itself will make the haemostasis very difficult, because the ligament is a firm structure which precludes adequate pressure application against the artery after angiography.

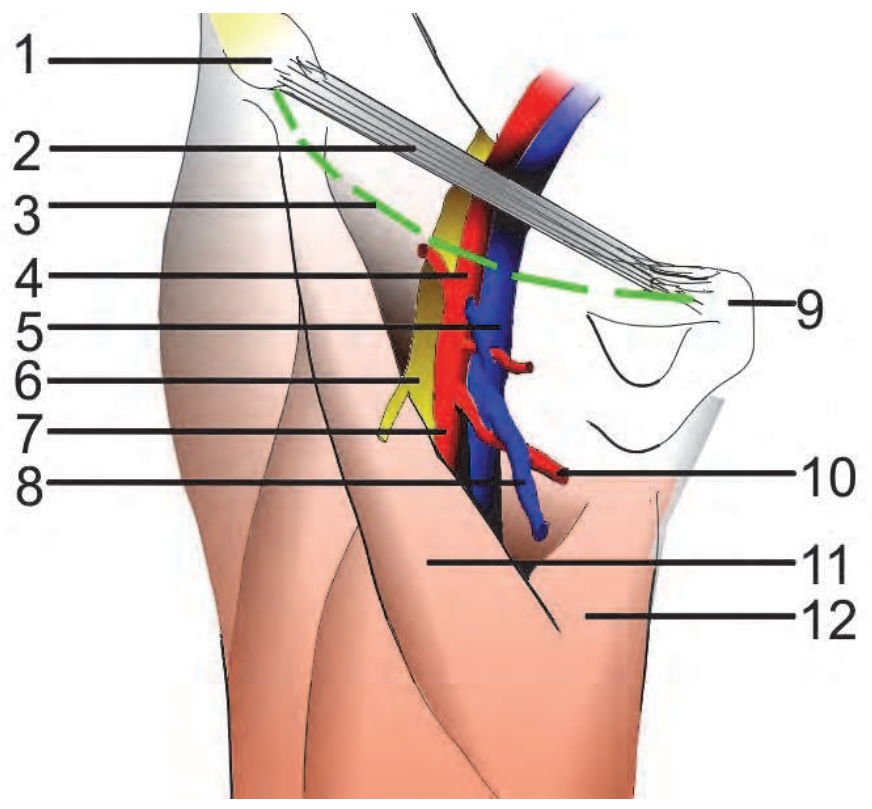

Fig. 10. Inguinal area anatomy; 1 - spina iliaca anterior superior, 2 - inguinal ligament, 3 skin crease, 4 - common femoral artery, 5 - common femoral vein, 6 - femoral nerve, 7 deep femoral artery, 8 - saphenous vein, 9 - pubic bone, 10 - superficial femoral artery, 11 sartorius muscle, 12 - abductor longus muscle 


\subsection{Radial approach}

Alternative access when femoral puncture is unavailable or cannot be used, is radial or brachial approach. While the femoral access is still most widely used in the USA and the most of Europe, Asia and Americas, radial access is becoming more and more popular in Europe, thanks to its advantage of avoiding prolonged periods of patients' bed-rest after the procedure. The transradial approach has gained popularity in recent years mainly because of the significantly decreased access-site complications and the greater patient comfort it offers (Tomassini et al, 2011). However, the prevalence of transradial approach for coronary procedures worldwide is still quite low and nowadays, in the United States, for example, the favourite strategy for bleeding prevention is mostly based on the adoption of new antithrombotic drugs (such as bivalirudin and fondaparinux) rather than on the selection of an alternative, safer vascular access route (Rigattieri et al, 2010). Also, radial approach is somewhat more difficult to master, and requires more training than the femoral one. The main setback is the small diameter of the vessel, and the fact that the radial artery is prone to spasm. Allen's test is also mandatory to determine the ability of the ulnar artery to take over the circulation to the hand in case of an irreversible damage to the radial artery. Another disadvantage of right radial approach is the considerable technical difficulty in evaluating left interior mammary arterial graft. Instead, the left radial approach can be used. The third reason because of which radial artery might not be used is the inability to use that particular artery as a potential free arterial bypass-graft, should the bypass-surgery be indicated. Also, there has been some controversy in radiation dose to the operator when using radial approach. There were reports of significantly higher radiation doses for the operator in comparison to femoral approach, especially during the learning phase. Before proceeding with transradial coronary angiography, it is wise to administer premedication in order to reduce the probability of radial artery spasm or thrombosis. Premedication cocktail containing nitroglycerin and verapamil has been documented to be effective in preventing radial spasms. However, verapamil is relatively contraindicated for some patients with left ventricular dysfunction, hypotension and bradycardia. Intra-arterial premedication with 100 $\mu \mathrm{g}$ of nitroglycerin and 3,000 units of heparin is effective in preventing radial spasms during transradial cardiac catheterization, according to Chen et al (2006). Some authors recommend an intra-arterial cocktail consisting of $5 \mathrm{mg}$ of verapamil plus $200 \mu \mathrm{g}$ nitroglycerine in $10 \mathrm{ml}$ of normal saline, and others (Pancholy et al, 2006) use $200 \mu \mathrm{g} / \mathrm{ml}$ of nitroglycerin solution injected on the lateral and medial aspect of the previous location of the radial artery pulsation, should the arterial spasm occur after administration of lidocaine for local anesthesia, and before radial cannulation. During transradial coronary angiography, the wire should always go smoothly. If the operator feels some resistance, a peripheral radial angiogram should be taken. There is a possibility that a radial loop with recurrent radial artery exists, and the operator will be forced to change the access site. Left radial approach can be used comfortably: it is possible to bend the patient's arm over the abdomen and perform the procedure. Left radial approach is also favoured when re-evaluating left interior mammary artery (LIMA) to left anterior descending coronary artery (LAD) bypass grafts. The transradial approach can be safely proposed for all patients, with a low conversion rate to transfemoral approach when an attempt on both radial arteries is considered first (Guédès et al, 2010). Instead of JL4 used in transfemoral approach, for an average sized patient, a JL3.5 can be used. The catheters should always be exchanged over a long wire, so 
that renegotiating the wire through this spasm-prone and often tortuous vessel can be avoided. Some population studies have shown that patients prefer radial access because of early ambulation (Cooper et al, 1999) and some cardiologists also strongly prefer it. However, one should bear in mind that the most important indication for PCI today is acute myocardial infarction. This important setting must be considered when discussing the preffered vascular access, because this is the primary circumstance in which PCI improves survival. It can be expected that difficulties with radial approach and conversion to femoral approach may delay time to reperfusion, jeoppardizing the lives of critically ill patients, especially those in cardiogenic shock, requiring intra-aortic balloon pump, for example. So the question is: with the increased use of radial approach, should it become the new standard of care, especially regarding emergency interventions for acute myocardial infarction, and can primary $\mathrm{PCI}(\mathrm{pPCI})$ be routinely performed through radial approach?And the answer is: No. As for the setting of acute myocardial infarction (AMI), Vorobcsuk et al (2009) performed a systematic review and meta-analysis of 12 trials comparing the radial versus the femoral approach. They found no significant difference in the time to reperfusion between the two groups but found access site failure when transradial approach was used, which contributed to longer procedure times (Jolly et al, 2009). Recent systematic reviews and meta-analyses of trials in the acute setting of STelevation AMI, where time is of the essence, have shown radial access to be associated with a 10\% crossover rate increasing "time to reperfusion" in the individual case (Vorobcsuk et al, 2009). Those trials usually excluded unstable patients in cardiogenic shock requiring additional treatment options, such as intra-aortic balloon pumps, central venous access or temporary pacing. These patients are automatically treated using femoral access as it is more convenient and appropriate to use one access site. In some circumstances these patients arrest on the table necessitating cardiopulmonary resuscitation, and femoral access is simply easier and less fiddly under difficult circumstances (Patterson \& Foale, 2011). Radial artery puncture can only be attempted once. If that attempt fails, the morbidity associated with access site crossover occurs, including the risk of radial artery occlusion, but also the need for double site post-procedural care (Stella et al, 1997). The radial approach therefore increases procedure times, not only because of the steep learning curve to this technically more challenging approach. Radial artery anomalies are also relatively common and are a cause of transradial procedure failure even for experienced radial operators (Lo et al, 2009). Even when operators achieve equal procedural times using radial and femoral approach, there is still significantly greater fluoroscopy time with radial approach PCI. Inevitably an increase in the irradiation of staff and patient follows (Brasselet et al, 2008). Femoral access is easier for training, not limited by anomalous radial artery anatomy, and allows for both left and right mammary arterial bypass graft evaluation. It is also convenient if a venous puncture is necessary for obtaining haemodynamic measurements. In these circumstances, an adjecent femoral vein puncture requires "same-site" postprocedural management. Surely this does not mean that the transradial technique should be dismissed as a cardiology fashion. It should be learned because it has certain advantages in diagnostic coronary angiography and elective PCI, it is associated with an earlier patient discharge, and can be applied in circumstances of severe aortoiliac disease which precludes the use of femoral approach. However, in primary PCI, the current enthusiasm for using transradial approach is not sufficiently justified in evidence, and especially not justified enough to dismiss the femoral route from current training programmes. 


\subsection{Brachial approach}

Brachial approach remains an option too, most commonly the right brachial approach. The brachial artery is the distal continuation of the axillary artery, and itself separates distally into ulnar and radial arteries. Percutaneous transbrachial approach is useful and safe alternative for performing coronary angiography as well as PCI. The advantage of the brachial approach as an alternative to the radial one is that brachial approach needs no special premedication, and can be performed using the exactly same equipment (arterial sheath and catheters), often even the larger-ones, including $7 \mathrm{Fr}$ size, although most operators usually use the shorter brachial sheaths. For brachial approach it is necessary that an arm-board be placed for the patient's arm and hand during the preparation of the patient for the procedure. The antecubital fossa should be prepped and draped, and the technique of local anesthesia, arterial puncture, sheath placement etc. is generally similar to the one used for femoral access. When puncturing the brachial artery, care must be taken not to puncture the posterior arterial wall too. The complication rate is relatively low, and in the large series has not exceeded 2\% (Gonzalez et al, 1991). Complications of this site of arterial access include: brachial artery thrombosis, hemmorhage, pseudoaneurysm formation, cellulitis, phlebitis, and median nerve traction with possible irreversible damage.

\subsection{Axillar approach}

Axillary artery cannulation is a useful technique for obtaining central arterial access in patients with severe peripheral vascular disease in the lower and upper extremities, although it is very rarely used today. The axillary artery, which is the direct continuation of the subclavian artery, courses from the outer margin of the first rib to the tendon of musculus teres major, where it becomes brachial artery. The lateral portion of the axillary artery is cannulated, just lateral to the head of musculus pectoralis minor. The patient's underarm must be shaved and prepped in sterile fashion. The arm is hyperabducted and externally rotated in order to access the artery (Gonzalez et al, 1991). After applying local anesthesia, a skin incision is made using a no. 11 blade, and through that incision, an 18-gauge needle is advanced until pulsatile backflow is obtained. Care must be taken to only perform a singlewall puncture. Then, a J-wire and thereafter a side-arm sheath-dilator are placed into the artery as with other accesses, and angiography can be conducted. After the procedure, the patient is advised not to use the arm for at least one day to avoid bleeding. Complications of axillary arterial cannulation include arterial thrombosis, brachial plexus injury, hemorrhage, and pseudoaneurysm formation.

\section{Performing the coronary angiography}

Through the sheath placed into peripheral artery through which the vascular access is enabled, and over the guidewire, the first coronary diagnostic catheter is introduced through the peripheral artery (Fig.11), and farther into the aorta. Care must be taken that the atraumatic J-tip of the guidewire precedes the catheter, as to avoid injury to the vascular endothelium. The J-wire is 0.035 or 0.038 inch in diameter. There are many different types of guidewires, different in length, structure, coatings, diameter, tip structure and shape, and materials, available for different diagnostic and interventional vascular procedures. The usual choice is the 0.035 inch wire with a $3 \mathrm{~mm}$ J-tip. For tortuous vessels, a hydrophilic wire, such as Terumo Glidewire, or Wholey Wire with soft, floppy tip may be used. Glidewire should not be used through the needle, because of the possibility to damage the 
hydrophilic coating. Also, when advancing the catheter, the proximal part of the J-wire has to be visible on the outside of the catheter and secured by the operator's hand (Fig.11), as to avoid embolization of the J-wire into the patient's circulation. That would require an emergency vascular surgical procedure to remove the J-wire from the patient's aorta. Under the X-ray guidance, the guidewire tip is advanced up to the ascending aorta, and into the aortic root. Holding it in place, the coronary diagnostic catheter is advanced over the guidewire until it reaches the ascending aorta.

After the catheter reaches the Valsalva sinus in the aorta, the guidewire is withdrawn leaving the catheter in place. The outside portion of the catheter is connected to a manifold (Fig.12). The manifold is on the other side connected to the syringe (or an automated injection-device) for radiocontrast application, and on its side arms to the saline infusion, to the transducer for invasive blood-pressure monitoring, and to a radiocontrast container from which radiocontrast is taken into the syringe. Through the manifold, the operator controls the contrast injection, blood pressure, injections of heparinized saline if necessary, etc. To ensure that there is no air or clot within the coronary catheter, a syringe is connected to the catheter to aspirate and discard at least some $5 \mathrm{ml}$ of blood. This is the approximate volume of the catheter lumen. Some operators instead just allow a small "bleed back" from the catheter, after which the catheter is connected to the three-port manifold.

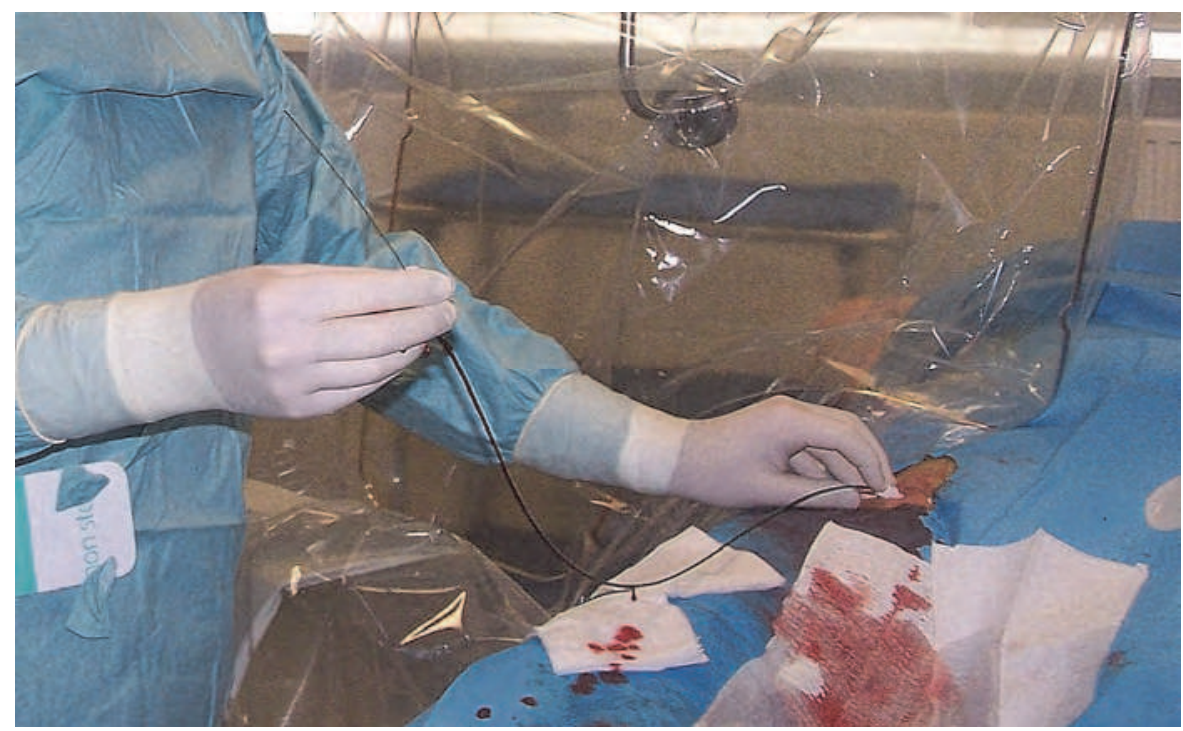

Fig. 11. Through the arterial sheath and over the guidewire, the first coronary diagnostic catheter is introduced into the artery; note the proximal end of the wire outside of the catheter

When connecting the catheter to the manifold, it is important to connect using the fluidagainst-fluid method, i.e. to let the blood back-bleed from the catheter, and at the same time to allow saline flush from the manifold. This manner of connecting the catheter to the manifold prevents air embolism from air bubbles from within the catheter, or from within the manifold. 


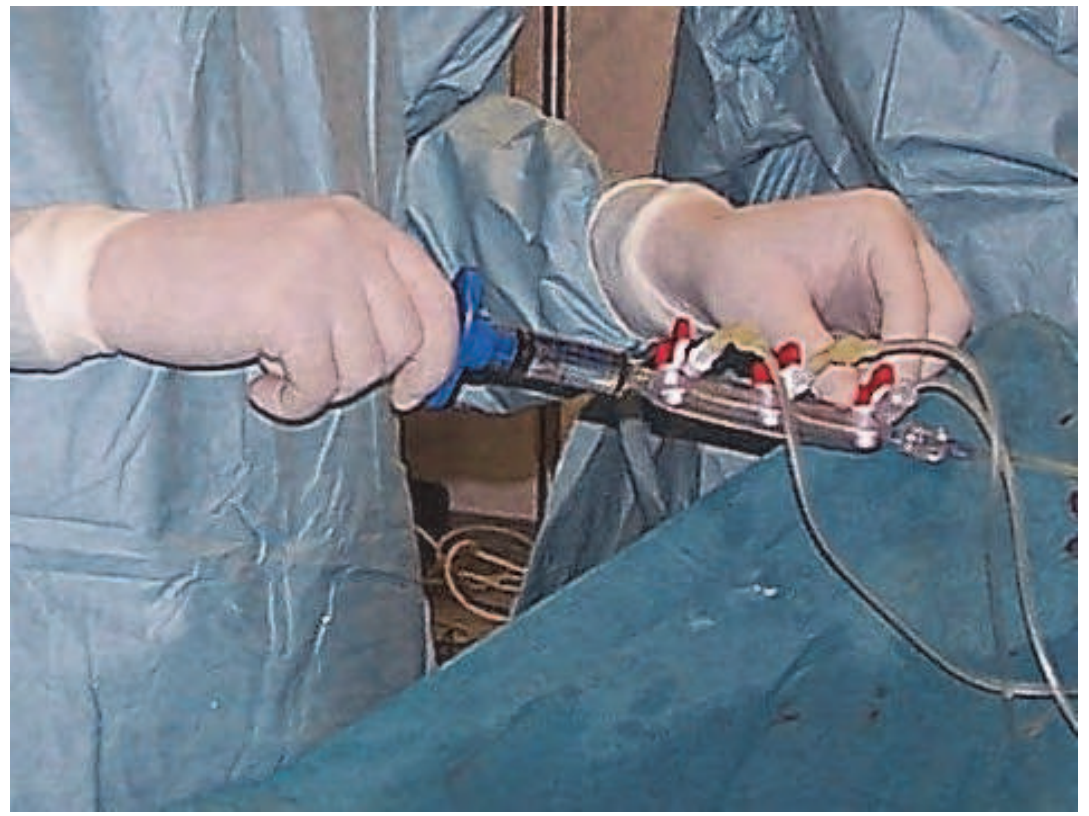

Fig. 12. Manifold with connections

\subsection{Left coronary artery engagement}

Under fluoroscopic guidance, the catheter is advanced into the coronary ostium, and several recordings of the left coronary artery are taken. The most commonly used catheter diameters are $6 \mathrm{Fr}$, but diameters from $4 \mathrm{Fr}$ to $8 \mathrm{Fr}$ can also be used. Diagnostic and therapeutic coronary catheters are pre-shaped to suite specific aortic and coronary anatomy. The most commonly used diagnostic catheters for coronary angiography are JL4 (Judkins left $4 \mathrm{~cm}$ curve) for the left, and JR4 for the right coronary artery. Both types of catheters were ingeniously designed by Judkins, and are easy to use, because they can engage the coronary ostia with very little manipulation. In fact, Judkins himself said that "no points are earned for coronary catheterization - the catheters know where to go if not thwarted by the operator" (Judkins, 1968). When cannulating the left coronary artery, the catheter is supposed to be unfolded in en face position in the ascending aorta. The best view to see the coronary ostia (both left and right) and to perform the ostial coronary cannulation is left anterior oblique (LAO) $50^{\circ}$, because sinus of Valsalva and aortic root are not superimposed on either of the coronary ostia. Cannulating the left coronary artery (LCA) in anteroposterior (AP) position is not recommended because it is difficult to appreciate the ostium, and ostial Left Main Coronary Artery (LMCA) stenoses can be easily missed.

For engagement of the LCA, the J-wire is advanced to the level of aortic valve. Then, the JL4 catheter is placed as low as possible facing the left coronary ostium. The guidewire is slowly withdrawn, and the catheter tip is already in the LMCA or just below it. If it is below the ostium, than it should be withdrawn ever so slightly, and it will fall into the LMCA ostium. Some operators fear that this method might be a bit risky, so they prefer to leave the catheter tip above the level of the left sinus of Valsalva, then withdraw the guidewire from the 
catheter, and thereafter advance the catheter tip downwards and into the LMCA ostium. This, on the other hand, carries some risk of scratching the surface of the aortic endothelium and embolizing atheromas if they are located in catheter's path. If the catheter tip is at the level of the ostium, but not within the ostium itself, it is usually easily inserted into the ostium by a clockwise or counter-clockwise torque. An injection of 5-10 ml of contrast close to the expected location of the coronary ostium reveals the position of the catheter tip within the aortic root and facilitates a precise cannulation. If the aorta is dilated, a JL5 or JL6 catheters may be required, while in small individuals, or with small aortic arches, a JL3.5 or even JL3 catheters may be used to cannulate the left coronary artery. Multiple recordings are taken from diffrerent perspectives. The optimal positions for recording the left coronary artery are discussed later in the chapter.

\subsection{Right coronary artery engagement}

After recording the luminograms of the left coronary artery, the catheter is removed over the J-wire, and another catheter, the one for the right coronary artery (RCA) is inserted through the sheath and into the aorta, up to the right coronary ostium, also in the LAO position. After reaching the ascending aorta with the tip of the right coronary catheter and removing the guidewire from inside of it, the catheter is aspirated and connected to manifold. The JR4 catheter is advanced into the aortic root, some $2 \mathrm{~cm}$ above the valve level. Then, the operator applies a clockwise rotation with some traction to the catheter. The catheter is slowly rotated using the rotation swivel at the distal portion of the manifold, where the coronary catheter is attached. As the catheter rotates, the torque should be reduced, so that there is no overshoot of the catheter over the right coronary ostium. The catheter rotates and descends somewhat, thereafter cannulating the right coronary ostium. If the first rotation is unsuccessful in cannulating the artery, the catheter should be rotated counter-clockwise to the initial position as to avoid the kinking of the catheter. The problem to find the coronary artery is most commonly due to the origin of the RCA superiorly and more leftwards than usual. It may even arise from the left coronary sinus, close to the LMCA. Using an Amplatz left (AL) 1 or 2 coronary catheter can be helpful to perform selective cannulation of such an RCA. Usually two, but sometimes more right coronary artery luminograms from different projections are recorded, and the catheter is removed. If the right coronary artery ostium is oriented straight downwards, a multipurpose (MP) catheter may be required. In contrast, if the ostium is oriented upwards, a "shepherd's crook" (SC) catheter or internal mammary (IM) catheter may be required. The optimal positions for recording the right coronary artery are discussed later in the chapter.

\subsection{Contrast injection technique}

The syringe with radiocontrast is held with the handle elevated, so that any air-bubbles inside of the syringe can rise to plunger, and are less likely to be injected into the patient's coronaries. A significant air-embolism into a coronary artery may result in asystole, and cardiac massage should be initiated. This usually reverts pretty quickly, as the air components of the bubble (nitrogen, oxygen, carbon-dioxide etc) are dissolved in the blood. Of course, the operator must previously make sure that there are no air-bubbles inside of the syringe. The radiocontrast must be injected vigorously enough to ensure that it temporarily 
replaces the blood in the coronaries, with a continuous back-flow into the aortic root. A weak injection may appear as a pulsatile contrast flow with filling-defects, and fail to display the coronary lumen adequately. It sometimes causes the operator to declare a coronary stenosis existence in a place where only a simple contrast filling defect exists. On the contrary, a too-forceful injection can, in extreme cases, cause a coronary dissection, although this event is extremely rare today. If there is no contrast back-flow to the aortic root, or if the back-flow is inadequate, there may be an ostial lesion present.

\subsection{Pressure damping}

During the cannulation of the coronary artery ostia, a special care must be taken to monitor the blood pressure. A sudden fall in blood pressure may lead to ventricular fibrillation and cardiac arrest. An immediate defibrillation usually restores the normal heart rhythm, although sometimes a full spectre of cardiopulmonary resuscitation has to take place, along with the cardiac massage, endotracheal intubation, artificial ventilation etc. This usually occurs if there is a severe ostial stenosis in the LMCA or the RCA, or if the artery is very prone to severe spasms, or if the conus branch is superselectively cannulated during the RCA engagement. It is a sign of a total coronary obstruction. Pressure damping or "ventricularization", called so because the pressure waveform resembles that of the ventricle, especially with very low diastolic pressure, can also be the result of placement of the catheter tip with the end-hole against the vessel wall, or presence of a thrombus, or an air bubble inside the catheter lumen, and sometimes also occurs after kinking the catheter body.

Whenever blood-pressure damping occurs during the coronary artery engagement, it is best to remove the catheter immediately (Conti et al, 1980). A repeated, careful cannulation may be attempted, best preceded by administration of nitroglycerin. If pressure damping occurs again, the angiogram can be swiftly recorded, and the operator must remove the catheter immediately. Sometimes, a non-selective or semi-selective opacification from the aortic root, with the catheter just in front of the coronary ostium may be sufficient to delineate the ostial coronary disease. There is also an option to use the side-hole catheters, which enable coronary perfusion through the sideholes located just proximally to the catheter tip.

\section{Coronary anatomy and the purposeful angiographic projections}

Since the coronary arteries are three-dimensional structures, and the X-ray image is twodimensional, several different perspectives of observing the coronary luminograms are necessary in order to adequately depict the coronary artery anatomy, the location, the extent, and the degree of coronary obstruction. The projections are defined according to the position of the digital flat panel detector, or image intensifier, depending on what kind of cardioangiograph is used. The X-ray tube is under the cardioangiograph table (Fig.1), i.e. under the patient. The positions can be defined as a combination of antero-posterior (AP), cranial, caudal, left or right lateral, and left or right anterior oblique (LAO and RAO, respectively). Extremely rarely, practically almost never, are posterior oblique positions used in diagnostic coronary angiography. The eleven regularly used positions at varying angles are: AP, AP-cranial, AP-caudal, left lateral, right lateral, RAO (right anterior oblique), 
RAO-cranial, RAO-caudal, LAO (left anterior oblique), LAO-cranial, and LAO-caudal. These positions are also defined by angles to cranial or caudal, and angles to the left or to the right. These angles may vary depending on patients' anatomy, coronary anatomy, position of the heart etc.

\subsection{Radiological coronary anatomy}

Left coronary artery is a complex structure, and demands usually at least 4-5 projections. In those, the Left Main (LM, or LMCA) coronary artery has to be clearly visible, along with its bifurcation and branches. The LMCA is a short artery, with variable length, usually bifurcating into the left anterior descending and left circumflex coronary artery. Sometimes LMCA can be very short, almost non-existant as such, in which case there is a "shotgunostium" of the left anterior descending (LAD) and the left circumflex (CX) coronary artery. The LMCA is rarely longer than $2 \mathrm{~cm}$.

The LAD passes down the anterior interventricular sulcus and usually extends around the heart's apex. It usually gives rise to two diagonal branches (D-1 and D-2), although they may also vary in number. Diagonal branches are positioned on the left anterolateral part of the heart's surface. The LAD also gives rise to septal perforators. The first one (1st septal) is usually the largest of all the perforators. It marks the border between the proximal and the mid portion of the LAD. The distal portion of the LAD is the distal 1/3 of the vessel, and has no specific landmark as to define it against the mid part. It is important to note the largest septal branch if the patient is to be subjected to alcohol septal ablation for hypertrophic obstructive cardiomyopathy.

The circumflex artery is positioned in the atrio-ventricular (AV) groove and is located on the left side of the heart. It gives rise to several atrial branches, but also to very important marginal branches. As they are positioned on the margo obtusus cordis, they are called the obtuse marginal (OM) branches. There are usually three of them (OM-1, OM-2, and OM-3), but their number can also vary individually. Marginal branches are important to define, because they are an available target for coronary bypassing if surgical revascularization is planned. The CX artery is usually anatomically non-dominant, meaning that it does not give rise to posterior lateral (PLA), nor posterior-descending (PDA) artery, which in that case originate from the right coronary artery (RCA). If the CX continues through the AV-groove and gives rise to PLA, while the PDA originates from RCA, than CX and RCA are codominant. If the CX gives rise to both PLA and PDA, while the RCA is small and terminates before bifurcating to PLA and PDA, than the CX is anatomically dominant. In this case, it is usually the largest artery in coronary circulation. If it is non-dominant, than the territories of blood supply of both LAD and RCA are larger and more significant than the CX one.

The right coronary artery (RCA) can vary in the position of its ostium. It gives rise to conusbranch, which supplies the right ventricular infundibulum. The second most proximal branch of the RCA is the sinoatrial node artery. The RCA continues on along the right-side $\mathrm{AV}$-sulcus giving rise to a few right-atrial branches. It also gives rise to one or two acute marginal branches, called so because they are positioned on the margo acutus cordis. They supply the right ventricular myocardium. Before reaching the crux cordis, the RCA also gives off a few posterior right ventricular branches on the right-diaphragmatic surface of the heart. Finally, at the crux cordis, on the heart's posterior wall, the RCA is bifurcating into the PLA and the PDA. This RCA dominance is the most common situation, present in some $85 \%$ 
of all human hearts. The PLA and the PDA have several smaller branches, e.g. the posterior septal perforators originating from the PDA. In the crux region, the atrioventricular node artery origins from the RCA, and passes vertically upward towards the AV-node. This is why ischemia in the region of RCA at the time of an acute inferior-wall myocardial infarction can be associated with different degrees of heart block.

\subsection{The best projections for evaluating the left coronary artery}

Left caudal view (LAO-caudal, or "spider" view) is excellent for evaluation of the LMCA and its bifurcation into the LAD and CX, or trifurcation into the LAD, CX and intermediate branch if it exists (Di Mario and Sutaria, 2005). Interventionalists use it in many cases for wiring the LAD or the CX. It forshortens the mid and the distal segment of the LAD, and should not be used to evaluate these segments, although it can pretty nicely separate LAD from diagonals and septals. Also, it can be very useful in viewing ostia of high marginal branches, and therefore is used for wiring these branches too. It is usually done in $25-40^{\circ}$ caudal and $30-50^{\circ}$ to the left.

Left cranial (LAO-cranial) view is very good for showing the ostium of the LMCA, but considerably forshortens the proximal half of the LAD. It is convenient for separating the diagonals and the septals from the LAD, though, and can be used to guide the guidewire into and from these branches during interventions. The $C X$ and its high-originating branches are not appreciated well in this projection, but if the CX is dominant, this projection can be useful to depict the very distal parts of the CX.

Cranial (AP-cranial) projection displays the ostium of the LMCA (Di Mario and Sutaria, 2005), similarly to the LAO-cranial view. Although some operators use it to intervene on the proximal LAD, the aouthor's opinion is that the overlap of the CX is too distracting, and that there are better projections for interventions and evaluation of the proximal LAD than the AP-cranial. This projection can be useful for evaluating the mid and distal segments of the LAD and diagonals' take-off, although the diagonal ostia can be overlapped with the maincourse LAD. The CX in this view often looks confusing for the inexperienced operator, especially in its proximal and mid segments, but if it is dominant, this can be a very useful projection for evaluating the very distal portions of the CX.

Caudal (AP-caudal) is a very good projection for evaluation of the LMCA, its bifurcation (or in some cases trifurcation), ostial LAD, and the intire CX if it is not dominant. This is the author's favourite projection for interventions on the CX. It can be useful for ostial stent placement in the LAD, similarly to LAO-caudal. Some operators prefere wiring of the marginal branches in RAO-caudal, but in that projection, the marginal ostia are often overlapped with the main course CX. The author feels that AP-caudal is the best projection for evaluating the $\mathrm{CX}$ in its entirety, including the marginal ostia, meaning that this projection is also probably the best for wiring those too. It is especially good for treating bifurcational lesions on $\mathrm{CX}+$ marginals, but in author's experience also good for treating bifurcation lesions on LMCA + LAD + CX, along with the spider-view. However, if the CX is anatomically dominant, the distal portions of the artery will usually overlap with 3rd marginal branch, PLA and/or PDA. For these, in the case of CX dominance, the cranial projections should be used.

Right cranial (RAO-cranial) projection is the most useful if it is performed with increased cranial angulation of $30^{\circ}$, or in some cases even more. This projection shows nicely the mid 
part of the LAD with the take-offs of diagonal branches and can in most instances be very useful for treating bifurcation lesions within the LAD + diagonals. Sometimes, the diagonal ostia will overlap, and LAO-cranial, LAO-caudal or AP-cranial will be better for that purpose. If the LMCA is very short, even proximal and ostial LAD can be nicely appreciated in steep RAO-cranial. The CX can be appreciated in this view only if it is dominant, and that goes only for its most distal parts. The proximal and the mid segment of the CX cannot be adequately evaluated in this view.

Right caudal (RAO-caudal) view is very good for evaluating the distal LAD. The proximal and the mid part of the LAD are overlapped with diagonals in most cases, so this should be taken into consideration when evaluating LAD in RAO-caudal. Still, some stenoses of the LAD can only be seen here, so it requires operator's attention. The LMCA is displayed in this view, although the bifurcation itself is being overlapped in most patients by the ostial segments of the LAD and the CX. The CX artery can be nicely evaluated in RAO-caudal, but to be sure, the AP-caudal should always be taken.

Left lateral is rarely used today. It can be useful to display LIMA-LAD anastomosis, the mid and the distal portions of the LAD, and to separate the LAD from the diagonals, LAD being the closest to the sternum. Pure RAO is rarely used today, since it does not display any of the LCA segments ideally, except it can be useful for evaluating the distal LAD. Pure LAO is very useful for cannulation of both LCA and RCA, but is not very useful in evaluating LCA or its branches, so it is not being routinely used for LCA today. Straight AP is also not an ideal projection for evaluation of the LCA, and for any of the segments, there are many more useful projections.

\subsection{The best projections for evaluating the right coronary artery}

Many operators believe that two projections can be quite enough for evaluation of the RCA since it has less complex anatomy in comparison to the LCA. The RCA can be viewed nicely in LAO, but even better in LAO-caudal, which is better than the former one in displaying the crux. The crux with the bifurcation of the RCA into the PLA and the PDA is also very nicely displayed in AP-cranial, and in some cases in RAO-cranial projection. Some operators use only simple LAO and RAO to evaluate the RCA. Left cranial (LAO-cranial) displays best the proximal and the distal RCA and is very good for displaying the crux. It can also be pretty good for displaying the PLA and the PDA (Di Mario and Sutaria, 2005). Cranial (APcranial), sometimes with just a small angulation to the right $\left(5-10^{\circ}\right)$ is very good for displaying the crux, the PLA and the PDA. The mid-RCA is best displayed in lateral and the RAO-view, but in most cases, LAO can be sufficient for this segment also.

\subsection{Angiographic studies and engagement of coronary bypass grafts}

When a previously operated patient, who had undergone a bypass-coronary revascularization, is subjected to coronary angiography, beside evaluating the native coronary arteries, a study of bypass-grafts is also necessary. The operator should read the surgical report in order to find out how many, and which bypass grafts there are. The venous, or free arterial bypass grafts, i.e. the bypass grafts for aorto-coronary bypassing are best cannulated from RAO $40^{\circ}$, but some operators cannulate them from LAO $50^{\circ}$, just as they do for native coronaries. Still, from RAO $40^{\circ}$ there is the best depiction of the grafts' origin from the aorta. The bypass graft for the RCA can usually be cannulated using the 
standard JR4, or JR3.5, sometimes JR3 catheter. The origin of the graft to the RCA is usually in the ascending aorta, just above the sinotubular junction. The cannulation is performed by placing the catheter high in the ascending aorta, applying clockwise-rotation, and, at the same time, pushing the catheter a bit downwards. If Judkins catheters are inadequate, RCB (right coronary bypass) catheter can also be used for cannulating the SVG (saphenous vein graft) to RCA.

The JR4 or JR3.5 catheters can also be used to cannulate all the other bypass grafts. Those grafts' origins are usually located higher in the ascending aorta, just beneath the arch. If the grafts for LAD, diagonals, intermediate, and obtuse marginals are unreachable with JR catheter, an LCB (left coronary bypass), or an AL catheter can be used. If grafts cannot be found selectively, an aortography in LAO position can be used to determine their origin, position, or stumps if they are occluded. For obtaining a proper evaluation, after aortography, a selective cannulation of the grafts should be reattempted.

Left internal mammary arterial graft can be cannulated using the IMA (internal mammary artery) catheter, but JR4, JR3.5, JR3, or short tip JR can also be used in certain situations. The ostium of the left internal mammary artery (LIMA) is engaged in $50^{\circ}$ LAO position, although sometimes it can be viewed better from AP or RAO $40^{\circ}$ position. First in $50^{\circ} \mathrm{LAO}$, a J-wire is passed into the left subclavian artery, beyond LIMA. Than the IMA catheter is passed over the wire, also beyond the LIMA origin. Then, from the position best suited for the operator $\left(\mathrm{AP}, \mathrm{LAO} 50^{\circ}\right.$, or $\mathrm{RAO} 40^{\circ}$ ), the catheter is slowly withdrawn, rotating the tip counterclockwise. Small test-injections are needed to display the ostium of the LIMA. The angiography should be performed from several positions, especially paying attention to the distal anastomosis with the LAD, since this position is the one most prone to iatrogenic injury from manipulation during the grafting procedure, thus being the most prone to developing a significant stenosis. A $90^{\circ}$ left-lateral position is almost mandatory in displaying LIMA-LAD graft, since it is very good to depict the distal anastomosis.

For cannulating the right interior mammary artery (RIMA), the brachicephalic artery is engaged, J-wire is advanced distally to the RIMA origin, from the right subclavian to the right axillary artery. The IMA catheter is advanced also distally over the wire. In the LAO $50^{\circ}$ projection, after removing the J-wire, the catheter is slowly withdrawn, and with occasional test-injections engaged into the RIMA ostium.

The right gastroepiploic artery has been used for coronary bypass, although very rarely. Surgeons sometimes use it when mammary arteries and saphenous vein grafts are unavailable. It is cannulated by first entering the hepatic artery using the cobra catheter. The operator must then advance a hidrophylic guidewire into the gastroduodenal artery, and then into the right gastroepiploic artery. Selective angiography of the letter will be enabled by switching the catheter, usually to right Judkins (Di Mario and Sutaria, 2005). The procedure is performed very rarely, and because of the unusual anatomy for the invasive cardiolgist, can be time-consuming.

\section{After the procedure}

The operator should always review his/her angiographic recordings before withdrawing the catheter, because standard projections used may not adequately reveal the condition of the coronary circulation, and additional shots may be necessary. The further therapeutic 
strategy should be considered (medical, surgical or interventional treatment). After the catheter is removed, and the procedure is finished, the patient is transfered out of the catheterization laboratory. Arterial sheath is removed, and manual pressure is applied onto the site of arterial puncture in order to stop the bleeding. That is followed by mechanical compression of that site in order to definitely stop the bleeding. The patient is later mobilized, and if there is no indication for the patient to stay in the hospital further, he/she is discharged on the next day, or sometimes on the same day after the procedure.

\subsection{Haemostasis}

Haemostasis is an important practical matter. Complications from diagnostic cardiac catheterization are rare, but if they happen, those related to haemostasis are the most common ones. When a patient comes out of the hospital after coronary angiography or coronary stenting, and gets a groin haematoma, he/she will most likely quickly forget the top-notch, several million euros costing equipment used during the procedure, as well as the skills of the operator who performed sometimes very difficult intervention, but will remember a large haematoma, and often complain about it to his family, neighbours, and friends. Therefore, it is very important to adequately perform haemostasis, not only for obvious medical, but also for "public-relation" reasons. The details of haemostasis and its possible complications are discussed elsewhere. However, there are a few points that need to be noted. Haemostasis may be difficult if the patient is obese, if there is high blood pressure (always try to regulate the blood pressure prior to sheath removal), or if clotting time is prolonged. If there is no vital indication, coronary angiography should be avoided if INR is $>1.6$, otherwise, the sheath should be sutured and removed upon achieving a more appropriate INR value. A significant aortic regurgitation with pulsus celer et altus can also make the post-sheath-removal hold difficult.

Large sheaths create bigger holes in the arterial wall, so the bigger the sheath diameter, the more difficult haemostasis will be. The patients who frequently elevate intra-abdominal pressure by sneezing, coughing or performing Valsalva maneuvers can prolong bleeding from the vascular access site. Same is true for restless patients, so those sometimes need to be sedated and observed by a nurse. In order to facilitate the haemostasis process, haemostatic devices have been developed, such as: collagen plugs, external pressure devices, vascular clips, external C clamps, topical haemostatic accelerators etc.

\section{Non-coronary left heart catheterization}

Sometimes, an additional catheter, called pigtail-catheter (because of its shape) is inserted into ascending aorta to perform aortography, and/or into the left ventricle to perform left ventriculography. Today, with skilled echocardiographers and top-notch cardiac ultrasound devices, these additional procedures are rarely used, but will be described too, so as to give the readers the necesary technical information about the value of these procedures, as well as how they should be performed.

\subsection{Left ventriculography}

Today, with skilled echocardiographers and excellent cardiac ultrasound equipment, there is far less need to perform left ventriculoghaphy than previously. With reliable 
echocardiograms, avoiding left ventriculography spares the patient from additional contrast administration, which is especially important if the patient already has impaired renal function. Also, without left ventriculography there is less radiation both for the patient and for the operator. If the patient is in heart failure or cardiogenic shock, every additional volume intake can prove to be risky for his/her condition. However, some operators and some centers prefer to perform the left ventriculography, especially if there is doubt about echocardiography findings, or if there is no echocardiography finding available.

In order to perform the left ventriculoghaphy, the operator advances the pigtail catheter over a J-wire to the aortic root. The author's choice is usually the angled version of the pigtail catheter. The J-wire is withdrawn into the catheter, with the J-wire tip just inside the catheter tip to add to the catheter stiffness in order to successfully pass over the aortic valve. The position of the digital flat-panel detector or the image intensifier is in RAO $30^{\circ}$. The catheter with the wire inside of it is advanced farther, so that the body of the catheter "bounces off" the aortic valve, and the distal curve faces upwards. Then the catheter is rotated in a clockwise fashion, being at the same time slowly withdrawn. This causes the catheter to rotate, and when the aortic valve opens, slip through the aortic ostium and into the left ventricle. If there are difficulties to pass the catheter into the left ventricle this way, the catheter tip is withdrawn into the ascending aorta, and the valve is probed with the Jwire. When the wire passes into the ventricular cavity, the catheter is advanced into the ventricle over the wire. Sometimes, a Glidewire with hydrophyllic coating must be used to pass the aortic valve, especially if there is a tight aortic stenosis. Also, sometimes it might be easier to pass the valve with a Judkins right coronary catheter.

The catheter is then held with the tip in the middle of the ventricle. The proximal end of the catheter is connected to the injector after it has been adequately aspirated and flushed. Through the automated injector, some $35-50 \mathrm{ml}$ of contrast is injected into the ventricle at the rate of $12-15 \mathrm{ml} / \mathrm{s}$, delineating the left ventricular cavity and displaying the left ventricle contractility. Intracavitary thrombi can be seen as hypodensities or filling-defects of contrast within the left ventricle. Mitral regurgitation, if exists, can be seen and semi-quantified, using degrees from 1 to 4 , with 4 being the most severe. Care must be taken when advancing the pigtail catheter into the left ventricle, because the mechanical contact with endocardium can provoke premature ventricular beats, even ventricular tachicardia. It can also provoke a complete heart block if the patient already has a right bundle-branch block, and the catheter irritates the left bundle-branch upon contact with left ventricular endocardium. If there are foreign masses within the left ventricle, such as thrombi or vegetations, those can be fragmented and embolized into systemic circulation, causing potentially catastrophic outcomes. A combination of a fragile myocardium and too vigorous catheter manipulation can cause myocardial rupture with fatal cardiac tamponade and refractory shock. Entanglement of the catheter into the chordae tendineae of the mitral valve or hooking the catheter tip on a mitral cusp can result in seemingly severe mitral regurgitation, so care must be taken to avoid this. In exceptional circumstances, catheter tip can theoretically rip a mitral cusp or break up a chorda or two, causing acute mitral regurgitation of various degree, thus complicating the left ventriculography.

After the ventriculography, the catheter is connected to manifold to record pressures in the left ventricle. Upon withdrawal of the catheter over the aortic valve, pressure curves in the aorta are also noted, and pressure values recorded. The systolic pressures in the aorta and 
the left ventricle are noted for transvalvular aortic pressure gradient. Care must be taken not to declare aortic stenosis in patients with previously unrecognized hypertrophic obstructive cardiomyopathy with high pressure-gradients across the left ventricular outflow tract, which may mimic the haemodynamics of aortic stenosis, with pressure gradients between the middle of the left ventricle and the ascending aorta.

Calculation of the left ventricular ejection fraction (LVEF) is usually performed using the software already incorporated into the imaging review station. Some operators use the semiquantitative eye-ball method in LVEF estimation, which has, when used by experienced operators, shown a surprisingly good correlation with the actual value of LVEF measured invasively in several studies. The software calculates LVEF by subtracting left ventricular end-systolic volume from the left ventricular end-diastolic volume.

Mastering the left ventriculography is important as the transfemoral - transcatheter aortic valve implantation is being performed increasingly. For severely stenosed aortic valves, it may be easier to pass the valve using an AL catheter and a Glidewire.

\subsection{Aortography}

Aortography is defined as a radiographic recording of injection of a large volume of radiocontrast into the aortic root. Most commonly, some $50 \mathrm{ml}$ of radiocontrast, at a rate of $15-25 \mathrm{ml} / \mathrm{sec}$ with a $0.5 \mathrm{sec}$ linear rise is used. The catheter of choice is a sidehole catheter, usually the pigtail shape. The best projection for estimation of aortic regurgitation is $\mathrm{LAO} 45^{\circ}$. Aortic regurgitation is semi-quantified and described in degrees from 1 to 4 , much like mitral regurgitation. If the patient is subjected to invasive diagnostics because of suspected coarctation of aorta, the coarctation will be best visible if aortography is performed in LAO $20^{\circ}$ position. Using the pressure monitoring, the gradient across the coarctation can also be measured. For evaluation of a suspected patent ductus arteriosus, the shot is usually taken at a very steep LAO projection. Aortography is usually done for evaluating the ascending aorta for dilation or aneurysm, and/or aortic regurgitation. The J-wire and the catheter are positioned in the ascending aorta, inside the root or at the level of sinotubular junction. Anatomy and dimensions of the ascending aorta and aortic arch are noted, as well as aortic regurgitation (if such a disorder exists), and/or aorto-coronary bypass grafts, also if they exist. Care must be taken that the catheter does not protrude back into the left ventricle during injection, which can make quantification of the aortic regurgitation difficult.

\section{Conclusion}

Invasive cardiologists today generally continue their education and become interventionalists. Anyone who wants to become a good interventionalist must be able to routinely perform diagnostic coronary angiography and to be able to solve the problems that might occur during the procedure. Therefore, the techniques of performing coronary angiography must be mastered by an invasive cardiologist. That creates the conditions for swift procedures, informative findings, purposefull conclusions and further recommendations, and last, but not least, the maximum safety for the patient. However, even with most carefully conducted coronary angiograms, there can be false readings. This is especially true for less experienced operators, who can sometimes misinterprete the 
finding, thus affecting the final recommendation for further treatment. These pitfalls may be: inadequate number of projections, ostial coronary occlusions without visible stumps, superselective injection, e.g. especially if LAD and CX have (almost) separate ostia, catheter induced coronary spasm, congenital coronary anomalies, pulsatile injection of radiocontrast with filling defects mimicking coronary atherosclerotic obstruction, myocardial bridging the key to recognize it is the fact that the lumen normalizes upon muscle relaxation, i.e. during diastole. Today, coronary angiogram projections must be observed by the operator in the context of future percutaneous coronary intervention. The lesions are not assessed only as significant or non-significant, but are evaluated regarding their length, their position regarding side-branches and other caracteristics important for the possible intervention. Therefore, it is of paramount importance to adequately display the coronary arteries and their lesions, in appropriate projections, with an adequate number of different views, still remaining rational regarding radiation and contrast medium consumption. Interventional procedures in cardiology today have expanded beyond the treatment of coronary artery disease. We are witnessing a technopharmacological improvement which enabled the interventional, catheter-based treatment of valvular heart disease, closure of interatrial and interventricular septal defects, relief of aortic coarctations, interventional treatment of hypertrophic obstructive cardiomyopathies, paravalvular leak closure, left atrial appendage closure, peripheral vascular disease treatment etc, and the basis for performing any of the interventional procedures, especially percutaneous coronary interventions, is mastering coronary angiography first.

\section{References}

Brasselet C, Blanpain T, Tassan-Mangina S, Deschildre A, Duval S, Vitry F, Gaillot-Petit N, Clément JP, Metz D. (2008) Comparison of operator radiation exposure with optimized radiation protection devices during coronary angiograms and ad hoc percutaneous coronary interventions by radial and femoral routes. Eur Heart J 2008 Jan;29(1), pp. 63-70, ISSN 0195-668x

Chen CW, Lin CL, Lin TK, Lin CD. (2006) A simple and effective regimen for prevention of radial artery spasm during coronary catheterization. Cardiology. 2006;105(1): pp. 437, ISSN 1878-5409

Conti CR, Levin DC, Grossman W. (1980). Coronary Angiography, In: Cardiac catheterization and angiography, Grossman W, pp. 147-169, Lea \& Febiger, ISBN 0-8121-0712-4, Philadelphia, Pa, USA

Cooper CJ, El-Shiekh RA, Cohen DJ, Blaesing L, Burket MW, Basu A, Moore JA. (1999). Effect of transradial access on quality of life and cost of cardiac catheterization: a randomized comparison. Am Heart J 1999 Sep;138(3 Pt 1): pp. 430-6, ISSN 00028703

Di Mario C \& Sutaria N. (2995). Coronary angiography in the angioplasty era: projections with a meaning. Heart 20052005;91: pp. 968-976, ISSN 1355-6037

Gonzalez A, Rahimtoola SH, Kulick DL. (1991). Technique of Vascular Access, In: Techniques and Applications in Interventional Cardiology, Kulick DL and Rahimtoola SH, pp. 1-18, MosbyYear Book, ISBN 0-8151-7048-3, St. Louis, MO, USA 
Guédès A, Dangoisse V, Gabriel L, Jamart J, Chenu P, Marchandise B, Schroeder E. (2010) Low rate of conversion to transfemoral approach when attempting both radial arteries for coronary angiography and percutaneous coronary intervention: a study of 1,826 consecutive procedures. J Invasive Cardiol. 2010 Sep;22(9): pp. 391-7, ISSN 1042-3931

Jolly SS, Amlani S, Hamon M, Yusuf S, Mehta SR. (2009). Radial versus femoral access for coronary angiography or intervention and the impact on major bleeding and ischemic events: a systematic review and meta-analysis of randomized trials. Am Heart J 2009 Jan;157(1): pp. 132-40, ISSN 0002-8703

Judkins MP: Percutaneous transfemoral selective coronary angiography. Radiol Clin North Am 61968 Dec;6(3): pp. 467-92, ISSN 0033-8389

Kwac MS, Yoon SJ, Oh SJ, Jeon DW, Kim DH, Yang JY. (2010). A rare case of radial arteriovenous fistula after coronary angiography. Korean Circ J. 2010 Dec;40(12): pp. 677-9, ISSN 1738-5520

Lo TS, Nolan J, Fountzopoulos E, Behan M, Butler R, Hetherington SL, Vijayalakshmi K, Rajagopal R, Fraser D, Zaman A, Hildick-Smith D. (2009). Radial artery anomaly and its influence on transradial coronary procedural outcome. Heart 2009;95: pp. 410-415, ISSN 1355-6037

Pancholy SB, Coppola J, Patel T. (2006). Subcutaneous administration of nitroglycerin to facilitate radial artery cannulation. Catheter Cardiovasc Interv. 2006 Sep;68(3): pp. 389-91, ISSN 1522-1946

Patterson T, Foale RA. (2011) If the radial artery is the new standard of care in primary percutaneous coronary intervention, why is most intervention done by the femoral approach? Heart 2011;97: pp. 521-522, ISSN 1355-6037

Powell D \& Moxey CF. (2011). Diagnostic Catheterization, In: Invasive Cardiology: a manual for cath lab personnel, 3rd Edition, Watson S \& Gorski KA, pp. 143-162, Jones \& Bartlett Learning, ISBN 978-0-7637-6468-5, Sudbury, MA, USA.

Rigattieri S, Di Russo C, Silvestri P, Fedele S, Loschiavo P. (2010). Our technique for transradial coronary angiography and interventions. Indian Heart J. 2010 MayJun;62(3): pp. 258-61. ISSN 0019-4832

Stella PR, Kiemeneij F, Laarman GJ, Odekerken D, Slagboom T, van der Wieken R. (1997). Incidence and outcome of radial artery occlusion following transradial artery coronary angioplasty. Cathet Cardiovasc Diagn 1997 Feb;40(2): pp. 156-8, ISSN 00986569

Tomassini F, Gagnor A, Varbella F. (2011). Successful use of an extra-long hydrophiliccoated sheath in enlarged aorta to overcome extreme tortuosity of right subclavian artery via transradial approach during coronary angiography. J Invasive Cardiol. 2011 Mar;23(3): pp. 56-7, ISSN 1042-3931

Vorobcsuk A, Konyi A, Aradi D, Horváth IG, Ungi I, Louvard Y, Komócsi A. (2009) Transradial versus transfemoral percutaneous coronary intervention in acute myocardial infarction: systematic overview and meta-analysis. Am Heart J 2009 Nov;158(5): pp. 814-21, ISSN 0002-8703 
Watson S \& Hunter J. (2011) Cath Lab Staff Duties, In: Invasive Cardiology: a manual for cath lab personnel, 3rd Edition, Watson S \& Gorski KA, pp. 119-129, Jones \& Bartlett Learning, ISBN 978-0-7637-6468-5, Sudbury, MA, USA. 


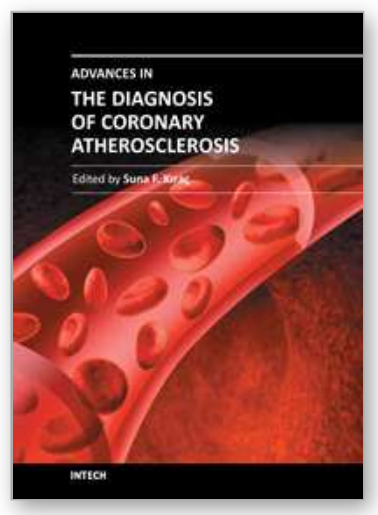

\author{
Advances in the Diagnosis of Coronary Atherosclerosis \\ Edited by Prof. Suna Kirac
}

ISBN 978-953-307-286-9

Hard cover, 378 pages

Publisher InTech

Published online 09, November, 2011

Published in print edition November, 2011

Coronary artery disease $(C A D)$ and its consequences are most important morbidity and mortality reasons in the developed and developing countries. To prevent hard end-points, early definitive diagnosis and optimum therapy play significant role. Novel advanced diagnostic tests which are biomarkers of inflammation, cell adhesion, cell activation and imaging techniques provide to get the best result in the detection and characterization of calcified or uncalcified atherosclerotic plaques. In spite of last developments in the imaging methods, coronary catheterization is still frequently performed. Following the first cardiac catheterization performed in 1844, date by date historical developments and the mechanics of cardiac catheterization techniques, risks associated with coronary angiography, and also, preventions and treatments of possible complications have been presented in this book. Other important issue is radiation exposure of patients and staff during coronary angiography and scintigraphy. Radiation dose reduction techniques, general radiation protection principles have been discussed in related chapters.

\title{
How to reference
}

In order to correctly reference this scholarly work, feel free to copy and paste the following:

Jasmin Caluk (2011). Procedural Techniques of Coronary Angiography, Advances in the Diagnosis of Coronary Atherosclerosis, Prof. Suna Kirac (Ed.), ISBN: 978-953-307-286-9, InTech, Available from: $\mathrm{http}: / / \mathrm{www}$.intechopen.com/books/advances-in-the-diagnosis-of-coronary-atherosclerosis/proceduraltechniques-of-coronary-angiography

\section{INTECH}

open science | open minds

\section{InTech Europe}

University Campus STeP Ri

Slavka Krautzeka 83/A

51000 Rijeka, Croatia

Phone: +385 (51) 770447

Fax: +385 (51) 686166

www.intechopen.com

\section{InTech China}

Unit 405, Office Block, Hotel Equatorial Shanghai

No.65, Yan An Road (West), Shanghai, 200040, China

中国上海市延安西路65号上海国际贵都大饭店办公楼405单元

Phone: +86-21-62489820

Fax: $+86-21-62489821$ 
(C) 2011 The Author(s). Licensee IntechOpen. This is an open access article distributed under the terms of the Creative Commons Attribution 3.0 License, which permits unrestricted use, distribution, and reproduction in any medium, provided the original work is properly cited. 